\title{
Level 3: Hardware - mächtige Gaming-Werkzeuge in der Bibliothek
}

\section{Einführung}

Wenn Sie sich dafür entschieden haben, etwas mit Computerspielen zu tun, dann geht es im nächsten Schritt um die Beschäftigung mit der Hard- und Software. Aus diesem Grund geht es nun um Konsolen, Computer, Smartphones, Tablet-PC’s, etc. Computerspiele zeichnen sich dadurch aus, dass man um sie zu spielen eine bestimmte Hardware benötigt. Für Sie als Bibliotheksmitarbeiter ist es wichtig zu verstehen, welche Hardware es gibt und vor allem was man damit machen kann. Sie müssen nicht der absolute Hardware-Profi sein. Sie werden niemals einen XBOX360 aufschrauben und reparieren müssen. Sie werden auch niemals in die Lage versetzt werden, einen Computer reparieren zu können. Trotzdem ist es wichtig, dass Sie verstehen, welche Hardware es gibt und wie sie funktioniert. Sie brauchen diese Informationen um entscheiden zu können, ob und wenn ja welche Hardware Sie für Ihre Bibliothek anschaffen. Wenn Sie sich mit dem Thema Gaming intensiv befassen möchten, dann werden Sie früher oder später eigene Hardware anschaffen müssen. Sie benötigen diese um z.B. eigene Events durchführen zu können. Manche Bibliotheken schaffen es, mit nur einer einzigen Konsole eine ganze Reihe von spannenden Veranstaltungen durchzuführen. Andere Bibliotheken haben Ihre Konsole an einem bestimmten Ort fest installiert und bieten ihren Nutzern/Kunden an, diese täglich zu benutzen. Wie eine solche Gaming-Station aussehen könnte, werde ich noch erklären. Das vielleicht wichtigste Argument für die Anschaffung einer eigenen Konsole (oder eines eigenen Gaming-PCs) ist, dass dann Ihr Team Gaming ausprobieren kann.

Für Ihr Team ist diese Erfahrung besonders wichtig. Früher oder später werden alle Teammitglieder in die Gaming-Aktivitäten Ihrer Bibliothek eingebunden werden. Dabei ist Gaming für viele Bibliotheksarbeiter ein völlig neues Thema. Neben manchmal vorhandener Abneigung gegenüber dem Thema an sich, bestehen sehr oft Berührungsängste. Man möchte nicht zeigen, dass man von dem Thema keine Ahnung hat. So entsteht in manchen Fällen ein doppelter Druck. Wenn Sie aber mit dem Thema starten, werden Ihre Mitarbeiter z. B. mit Fragen zur Hardware konfrontiert werden. In diesem Fall ist es gut, wenn Ihr Team zumindest grundsätzlich weiß, wie die Systeme funktionieren. Die Nutzung von verschiedenen Konsolen ist in ihren Kernpunkten nahezu vergleichbar. Es gibt zwar technologische Unterschiede, wenn Sie aber eine der Konsolen und/oder die Nutzung eines Gaming-PCs verstanden haben, wird das Verständnis von anderen Modellen umso leichter fallen.

Wie Sie bereits in der Beschäftigung mit der Geschichte des Gamings lernen konnten, begann der Siegeszug des Gamings mit der Einführung der Spielkonsole „Odyssee“ des Unternehmens Magnavox. Diese Konsole war sehr einfach. Die Grafik war rudimentär und man konnte nur gegeneinander antreten. Das System war nicht in der Lage, ein komplexes Spiel, darzustellen. Die Erfolgsgeschichte der Odyssee basiert mit Sicherheit auch auf der Tatsache, dass sie quasi eine Funktionserweiterung des Fernsehens darstellte. Somit war Sie von vornherein an das zentrale Massenmedium der damaligen Zeit gekoppelt.

Das Grundmuster ist bis heute gleich geblieben. Es gibt die Zentraleinheit, die das Spiel berechnet (Konsole, PC, Smartphone, Tablet-PC, etc.). Sie ist die zentrale Einheit. Die jeweiligen Spiele werden in die Zentraleinheit geladen. Dies kann durch physische Datenträger oder heute vermehrt durch Downloads geschehen. Die Zentraleinheit ist an einen Monitor/TV angeschlossen. Auf diesem Monitor wird das Spiel 
dargestellt. Das Spiel wird mittels Controllern gesteuert, welche an die Zentraleinheit angeschlossen werden. Das alles klingt mit Sicherheit etwas abstrakt, aber es sind letztlich immer vier Einzelteile: Zentraleinheit, Spiele, Controller, Monitor bzw. Ausgabegerät. Im Laufe der Jahre hat sich dieses Modell in Teilen verändert. Die Spielautomaten, welche noch heute in manchen Spielhallen zu finden sind, haben alle vier Elemente in einer Maschine vereint. Gleiches gilt für die Smartphones und Tablet-PCs. Des Weiteren haben sich die Controller weiterentwickelt. Besonders interessant ist hierbei das Kinect-System, welches Bestandteil der XBOX360 ist und auch der kommenden XBOX One sein wird. Dabei handelt es sich um ein Kamerasystem, welches den gesamten Körper des Spielers zum Controller werden lässt.

Die Bereiche Hard- und Software sind zwar ein wichtiger Bestandteil dieses Buches. Jedoch möchte ich nochmal darauf hinweisen, dass es hier in sehr kurzer Zeit zu großen Veränderungen kommt. So wird vor der Veröffentlichung dieses Buches eine neue Konsolengeneration auf den Markt kommen. Es handelt sich dabei um die Playstation 4 und die XBOX One. Leider ist es mir nicht möglich gewesen, vorab eine Playstation 4 zum Testen zu bekommen. Zudem gibt es mit dem Unternehmen „Valve“ demnächst einen weiteren interessanten Anbieter von einer Gaming-Konsole. Natürlich sollen Sie aber auch zu diesen Geräten Informationen bekommen. Deshalb können Sie auf www.christoph-deeg.de ein Update zu diesem Teil des Buches finden.

Wenn man sich nun mit der Hardware im Detail beschäftigt, steht man vor einem kleinen Problem. Welche Hardware sollte man nun beschreiben? Welche Systeme sind für Bibliotheken relevant? Auf den ersten Blick erscheint die Antwort ganz einfach: man nimmt die Angebote, welche aktuell erfolgreich sind und bei denen man deshalb davon ausgehen kann, dass es dazu eine Nachfrage geben wird. Und natürlich werden nun die aktuellen Systeme beschrieben werden. Ich bin jedoch zu der Überzeugung gelangt, dass dies nicht ausreicht. Es ist m.E. ebenso wichtig, dass Sie auch ältere Modelle bzw. Nischen kennenlernen. Dies ist vor allem im Bereich „Retrogaming“ interessant. Bei meinen Besuchen in den USA und Asien habe ich erleben dürfen, dass sehr viele Menschen nicht nur an den aktuellen Spielen und damit verbunden der aktuellen Hardware interessiert sind. Ebenso spannend erscheint das Spielen mit alten System wie z. B. dem Nintendo NES oder einem alten Computer wie dem C64. Spiele wie „Donkey Kong“, „The Legend of Zelda“, „Pac-Man“ ja selbst „Pong“ sind immer wieder ein interessantes Thema. Aus diesem Grund beschreibe ich neben den aktuellen Systemen auch einige wenige, die nicht mehr verkauft werden, die aber gleichzeitig eine Bedeutung für Bibliotheken haben könnten.

\section{Gaming-PCs}

Beginnen wir mit den Computern. Computer haben im Bereich Gaming noch immer eine sehr große Bedeutung. Vor allem in Deutschland gibt es eine große Fangruppe. Es sind übrigens vor allem die Computer und nicht die Konsolen, die für die massenhafte Verbreitung von Computerspielen auch in älteren Zielgruppen gesorgt haben. Denn es waren und sind Spiele wie Solitär und Mahjongg etc. die dafür sorgen, dass es mehr weibliche Gamer über 55 als männliche Gamer im Teenager-Alter gibt. In den meisten Büros stehen heute Computer und alle diese Systeme sind technisch in der Lage, Gaming zu ermöglichen. Wenn wir uns also über Computer und Gaming Gedanken machen, müssen wir diesen Bereich in zwei Teile teilen: Standard Computer für Spiele, die keine große Grafik- und Spielberechnung benötigen und spezielle GamingPCs für aufwendige Spiele. Grundsätzlich sind beide Systeme für Bibliotheken relevant und interessant. 
Ein Computer ist ein System, welches Sie mit Sicherheit kennen. Insofern möchte ich Ihnen jetzt nicht das Grundmodell eines PCs erklären. Wir haben den eigentlichen PC mit Mainboard, Prozessor, Speicher, Grafikkarte, Festplatte etc. Hinzu kommt ein Eingabegerät z. B. eine Tastatur und eine Maus sowie ein Monitor und gegebenenfalls Lautsprecher etc. Wenn wir uns nun mit Gaming-PCs beschäftigen, geht es um die gleichen Komponenten - nur dass es sich nun um Profi-Komponenten handelt. Ein Gaming-PC ist also ein ganz normaler PC mit Profi-Hardware. Wesentliches Kriterium ist die Grafikkarte. Normale PCs müssen selten komplexe Grafiken berechnen. Das Abspielen eines Films, das Surfen im Internet die Arbeit mit Office-Programmen, als dies erfordert in der Regel keine Hochtechnologie. Sollen aber Filme geschnitten oder komplexe Grafiken berechnet werden, werden hochwertigere Bauteile benötigt. Bei einem Gaming-PC ist die Grafikkarte der Bereich, der über die Qualität der Grafik des Spiels entscheidet. Hier wird berechnet, was der Spieler später sieht. Und dabei geht es sehr oft um die Darstellung von komplexen Details. So kostet eine vernünftige Gamer-Grafikkarte gerne einige hundert Euro. Diese Karten sind sehr groß und verbrauchen zumeist auch sehr viel Strom. Sie werden sehr heiß, wodurch sie in der Regel sehr große Speziallüfter haben. Sie verfügen über einen großen Videospeicher und benötigen eine bessere Stromversorgung. Die Grafikkarte alleine reicht aber nicht aus. Erst das Zusammenspiel aller Komponenten eines Gaming-PCs entscheidet über die Qualität des PCs. Konkret bedeutet dies, dass Sie ein gutes Motherboard, einen guten Prozessor, ausreichend Arbeitsspeicher, schnelle Festplatten (gerne SSD) und natürlich eine gute Grafikkarte benötigen.

Ist der PC erstmal gekauft geht es im zweiten Schritt um die Auswahl der Peripherie. Hierzu gehört die Tastatur, die Maus, eventuell weitere Controller, Lautsprecher und natürlich einen Monitor. Für alle diese Bereiche können Sie theoretisch auf Standartmaterial zurückgreifen. Jedoch reicht dies in der Regel nicht aus. Ich empfehle deshalb, auf speziell für Gamer entwickelte Hardware zurück zu greifen. Diese Hardware ist nicht so teuer wie man glauben mag. In vielen Büros werden zum Beispiel Tastaturen verwendet, die ein längeres Arbeiten am PC ohne körperliche Schäden wie z.B. Verkrampfungen etc. ermöglichen sollen. Solche Tastaturen kosten mehr als 10,- $€$ :-) Eine Gaming-Tastatur, die ich sehr oft nutze ist die Sidewinder X4 von Microsoft. Sie kostet zum jetzigen Zeitpunkt um die 40,- $€$.

Der große Unterschied sind die zusätzlichen Funktionstasten. Mit ihnen lassen sich sogenannte Makros programmieren. Makros sind individuell programmierte Tastenkombinationen. Um zu verstehen, warum dies so wichtig ist, muss man verstehen, wie Gamer Ihre Tastaturen nutzen. Grundsätzlich wird eine Tastatur genauso benutzt wie jede andere Tastatur auch. Man kann mit Gaming-Tastaturen ganz normal schreiben etc. Wenn man nun ein Spiel spielt, ändert sich die Nutzung der Tastatur nachhaltig. Im Folgenden möchte ich dies kurz erklären:

In der Regel nutzen die Spieler für die Bewegung ihrer Figuren eine Kombination aus Maus und Tastatur. Mit der linken Hand steuern sie durch die Tasten W,A,S,D die Vorwärts-, Rückwärts- und Seitwärtsbewegung der Spielfigur. Die Maus dient zur Festlegung der Richtung. Auf diese Art und Weise lassen sich Figuren in der FirstPerson und Third-Person-Ansicht sehr gut steuern. In anderen Spielen können Einheiten/Spielfiguren durch die Maus aktiviert bzw. gekennzeichnet um dann weitere Befehle über die Maus oder die Tastatur zu geben. Damit geht der Tastenwahnsinn aber erst richtig los. Natürlich werden alle Tasten einer Maus und sehr viele Tasten einer Tastatur genutzt. Mit den normalen Tasten einer Maus werden z. B. Gegenstände bewegt. Das spannende sind aber die Tastaturbelegungen und Sondertasten. Es gibt in vielen Spielen sehr viele Befehle, die man eingeben kann. Hierfür gibt es eine Vielzahl an Tastaturkürzeln. Es ist ein bisschen vergleichbar mit den Tastaturkombi- 
nationen beispielsweise in Office-Anwendungen. Nur das diese Kombinationen sehr oft und sehr schnell genutzt werden. Manche Tastaturen und Mäuse erlauben eine eigenständige Programmierung von Sondertasten. Diese Kombinationen nennt man Makros - in manchen Spielen vor allem in Onlinespielen sind diese Makros aber oft nicht erlaubt.

Die Tastaturen und Mäuse für Gamer müssen also so gestaltet werden, dass man damit lange spielen kann. Die Tasten müssen sehr schnell reagieren. Die Maus braucht eine sehr hohe und wenn möglich auch verstellbare Auflösung. Dies alles führt dazu, dass diese Geräte letztlich auch für den normalen Anwender attraktiv sein können. Ich selber nutze auch für die normale Officearbeit Gaming-Hardware. Nebenbei bemerkt: auch die Kosten für eine Gaming-Maus sind nicht zwangsweise sehr hoch. Für bereits 25,- $€$ bekommt man eine vernünftige Einsteiger-Maus.

Überlegen Sie bevor Sie einen neuen Gaming-PC anschaffen, ob sie diesen wirklich benötigen. Für sehr viele Spiele wird kein High-End-PC benötigt. Entwickeln Sie vor der Kaufentscheidung ein Portfolio für Spiele, die für Sie überhaupt infrage kommen. Klären Sie dann ab, welche Systemvoraussetzungen für die Spiele benötigt werden. Überprüfen Sie nun, ob Sie diese Spiele nicht auch auf Konsolen spielen können. Erst dann würde ich überlegen, ob man einen Gaming-PC benötigt.

Kaufen Sie keinen Komplettrechner. Diese All-Inclusive-Angebote sind in der Regel teurer und sehr oft auch weniger leistungsfähig als ein preislich vergleichbares selbst zusammengestelltes System. Es gibt eine große Zahl an PC-Händlern, die Ihnen anbieten, dass Sie die einzelnen Komponenten selber aussuchen und dann der PC von dem jeweiligen Händler zusammengebaut wird. Wenn Sie sich die Auswahl der einzelnen Komponenten nicht zutrauen, können Sie auch Magazine wie „Gamestar“ oder „PCGamesHardware“ lesen. Beide Magazine erstellen kontinuierlich neue Empfehlungen für sinnvolle Konfigurationen. Viele Bibliotheken müssen sich an ganz spezielle Ausschreibungsformalitäten halten. In diesen Fällen können Sie die Konfiguration ausschreiben und sich dann Angebote für Einzelteile, den Zusammenbau und die Einrichtung zukommen lassen. Einen nutzbaren Gaming-PC bekommen Sie schon für 600,- $€$ Nach oben sind natürlich keine Grenzen gesetzt ...

Achten Sie immer darauf, bei einem Gaming-PC kontinuierlich die Treiber zu aktualisieren. Vor allem für die Grafikkarte ist dies wichtig. Es gibt aktuell zwei relevante Anbieter von Gaming-fähigen Grafikkarten: AMD und NVDIA. Beide Anbieter entwickeln kontinuierlich neue Treiber um die Performance ihrer Hardware zu verbessern. Am einfachsten ist es wenn Sie sich eine automatische Benachrichtigungsmail zukommen lassen, die auf neue Treiber hinweist. Dieser Service ist kostenlos. Achten Sie am Anfang unbedingt auf Ihren Spamordner. Wenn dann eine Mail mit einer Benachrichtigung kommt, können Sie den Treiber runterladen. Hierfür müsste Ihnen u.U. von Ihrer IT-Abteilung eine spezielle Genehmigung eingerichtet werden. Alternativ kann die IT-Abteilung auch selber die Updates vornehmen.

Sie können bei Fragen nach der richtigen Hardwarekonfiguration auch in verschiedenen Online-Foren der Gamer-Community fragen. Eine Liste von interessanten Foren finden Sie u. a. auf meinem Blog www.christoph-deeg.de

\section{Stationäre Gaming-Konsolen}

Kommen wir nun zu den Konsolen. Konsolen waren die ersten erfolgreichen GamingMaschinen. Mit Ihnen wurden die Nutzungsmöglichkeiten des TV erweitert. Man spielte zuhause und man spielte zu zweit. Die „Odyssee“ von Magnavox haben Sie 
ja bereits kennengelernt. In diesem Abschnitt geht es um die Frage, was Konsolen überhaupt sind, was man damit machen kann und wie Bibliotheken damit umgehen sollten.

Zum jetzigen Zeitpunkt gibt es vier relevante stationäre Konsolen:

1. Die Nintendo Wii - ist noch immer in sehr vielen Haushalten vorhanden

2. Die Nintendo Wii U - diese Konsole ist noch verhältnismäßig neu und ersetzt die Wii

3. Die XBOX360 inkl. Kinect - diese Konsole wird durch die XBOX One ersetzt und ist ebenfalls weit verbreitet

4. Die Playstation 3 - auch diese Konsole wird einen Nachfolger nämlich die Playstation 4 ersetzt werden. Sie ist aber ebenfalls in vielen Haushalten vorhanden.

Grundsätzlich sind und waren Gaming-Konsolen dafür da, mit ihnen Computerspiele zu spielen. Diese Kernfunktion haben Sie noch heute inne. In den letzten Jahren hat sich aber die Funktion der Konsolen erweitert. Auf diesen Punkt werde ich später noch eingehen. Konsolen sind also Gaming-Maschinen. Eigentlich sind sie kleine Computer. Wenn man eine Gaming-Konsole öffnet, findet man die gleichen Bauteile wie in einem normalen Computer. Und wie bei einem normalen Computer benötige ich eben der Konsole noch einen Monitor sowie einen oder mehrere Controller. Der Vorteil von Gaming-Konsolen ist der, dass sie speziell für Games entwickelt wurden. Ihre Bauteile sind optimiert für das Spielen und die darauf spielbaren Games werden wiederum genau für die jeweilige Konsole entwickelt. Wir haben es also mit aufeinander abgestimmten Systemen zu tun. Für manche Spiele werden verschiedenen Versionen entwickelt. Diese Versionen sind an die jeweilige Hardware angepasst. So gibt es dann z. B. eine Version für die XBOX360, eine Version für die Playstation 4 und eine Version für den PC.

Man kann auf Konsolen alleine oder zusammen mit anderen Gamern spielen. Möchte man mit anderen zusammen spielen, gibt es zwei Möglichkeiten:

1. Die Spieler teilen sich die Konsole. Jeder Spieler hat einen Controller, der an die Konsole angeschlossen worden ist. Auf dem Monitor sieht man entweder einen sogenannten „split screen“, bei dem jeder Spieler seine Figur auf einem Teil des Monitors sieht, oder man spielt gemeinsam im gleichen virtuellen Raum. In diesem Fall sind alle Spielfiguren auf dem Monitor in einem Bild sichtbar.

2. Die Spieler sind online miteinander vernetzt. In diesem Fall spielt jeder Spieler an einer eigenen Konsole. Die Konsolen sind online miteinander verbunden. Der Vorteil dieser Variante ist der, dass auch Spieler miteinander spielen können, die sich nicht am gleichen Ort befinden. Dafür benötigt man allerdings einen kontinuierlichen Onlinezugang.

Wenn Sie also mit einer Konsole arbeiten möchten benötigen Sie folgende Ressourcen:

- Konsole

- Controller

- Spiele

- Strom für Konsole und Monitor

- Kabel für Verbindung mit Monitor (Wenn möglich HDMI)

- Monitor (mindestens 24“)

- Platz

- Möbel um die „Gamingstation“ aufzubauen (Tisch etc.) 
- Internetzugang

- Lautsprecher

- Ersatzbatterien - außer bei Playstation 3 - (am besten Akkus nebst Ladestation)

Bei allen aktuellen Modellen (Nintendo WII, Nintendo Wii U, Playstation 3 und XBOX360) benötigen Sie einen Onlineaccount. Zudem benötigen alle drei Systeme einen Zugang zum Internet. Über diesen Zugang werden u.a. die Systemupdates installiert, Zudem werden Informationen zu Spielständen etc. online gespeichert. Der Internetzugang kann bei allen vier Modellen über WLAN erfolgen.

Leihen Sie sich vor dem Kauf einer Konsole ein System aus und testen Sie damit, ob Sie mit dem WLAN bzw. dem LAN in Ihrer Bibliothek arbeiten können oder nicht. Es reicht nicht aus, dass Sie mit dem WLAN oder LAN auf das Internet zugreifen können. In vielen Fällen habe ich erlebt, dass man über das LAN und/oder WLAN der Bibliothek auf das Internet zugreifen konnte, jedoch nicht auf Plattformen wie z. B. das „Playstation Network“. Somit kann die Konsole dann nicht angemeldet und auch keine Updates runtergeladen werden. Einige Spiele sind dann u.U. nicht mehr spielbar. Zwar könnte man diese Konsole 2-3x in der Woche mit nach Hause nehmen, um dort die so wichtigsten Updates aufzuspielen, jedoch ist dies mit erheblichem zeitlichen Aufwand verbunden. Testen Sie zudem unbedingt die Bandbreite Ihres Netzes. Hierfür können Sie einfach 20 Kunden, die ein Smartphone oder einen Tablet-PC besitzen in die Bibliothek bitten, damit diese Ihre Geräte zum gleichen Zeitpunkt über Ihr WLAN mit dem Internet verbinden. Sollte dies Schwierigkeiten bereiten, sollten Sie unbedingt mit Ihrem Anbieter sprechen.

Planen Sie jede Woche etwas Zeit zum Testen der Systeme ein. Führen Sie kontinuierlich alle Systemupdates durch. Testen Sie, ob die Batterien der Controller geladen sind bzw. laden Sie die Controller der Playstation auf. Testen Sie auch den Monitor sowie alle Kabel.

Treffen Sie sich in Ihrem Team, um gemeinsam die Konsole auf- und abzubauen. So bekommen Sie schnell die notwendige Routine. Jedes Teammitglied sollte in der Lage sein, ein System auf- und abzubauen.

Sorgen Sie dafür, dass Sie immer genügend Ersatzteile (Kabel, Controller, Akkus etc.) vorrätig haben.

\section{Stationäre Konsolen als Multimediacenter}

Stationäre Gaming-Konsolen sind nicht nur Plattformen, auf denen man Computerspiele spielen kann. Sie sind vielmehr multioptionale Medienplattformen. Das bedeutet, dass man Sie auch für den Konsum anderer Medien wie z.B. Videos und Musik nutzen kann. Dabei stehen zwei Varianten zu Verfügung:

1. Abspielen von Mediendateien über CD's, DVD's, Blue-Rays, der internen Festplatte sowie einem externen Mediaplayer.

2. Abspielen von Medien mittels Streamingdiensten. In dieser Variante fungiert die Konsole als Zugang zu den Onlinediensten wie z. B. Lovefilm, Windows Live, Playstation Network, Watch ever, Sky Go

Mit diesen Funktionen sind diese Konsolen letztlich auch Konkurrenten zur den Verleihangeboten der Bibliotheken geworden. Auch der Kauf und der Download von Spielen direkt auf die jeweilige Konsole sind möglich. Dies hat einen nachhaltigen Einfluss auf die Bestandsstrategie einer Bibliothek. Dazu werde ich noch an anderer Stelle schreiben. 


\section{Konsolen als Kommunikationssysteme}

Nicht nur das Spielen von Computerspielen und der Zugriff auf Videos, Musik und das Internet ist mit den Konsolen möglich. Sie sind zudem Kommunikationsplattformen. Über sie kann der Nutzer auf eine Vielzahl an Communities zugreifen. Spieler können Sich untereinander austauschen indem sie z. B. chatten oder Teil einer Community werden. Unabhängig davon kann man bei einigen Spielen direkt aus dem Spiel Spielstände oder High-Scores auf Twitter oder Facebook posten.

\section{Welche Konsolen gibt es?}

Im Folgenden möchte ich die aktuell relevanten stationären Konsolen und ihre Besonderheiten beschreiben:

\section{XBOX360}

Anbieter: Microsoft

Veröffentlichung in Europa: 02.12.2005

Anschlüsse: Strom, USB 2.0. HDMI, AV-Ausgang, Ethernet (LAN), Optischer Digitalausgang

Sonstiges: WLAN, integrierte Festplatte oder kleiner Flash-Speicher

Die XBOX 360 ist der Nachfolger der XBOX. Ende 2013 wurde sie durch die XBOX One ersetzt. Die XBOX360 ist eine Konsole mit sehr guten Grafikeigenschaften. Für Bibliotheken ist vor allem das „Kinect“-System interessant - nähere Informationen zu diesem System finden Sie unter dem Abschnitt: „Controller“. Die XBOX360 ist eine multioptionale Medienplattform. Dazu gehört nicht nur die Möglichkeit, verschiedenen Medien abzuspielen bzw. den Zugang dazu zu bekommen. Mit der Gestensteuerung „Kinect“ kann die XBOX bequem vom Sofa aus mittels einfacher Gesten sowie einer Sprachsteuerung kontrolliert werden. Mit „Smart Glass“ kann zudem die XBOX über ein Smartphone oder ein Tablet gesteuert werden. In manchen Fällen werden über die mobilen Devices teilweise interaktive Zusatzinhalte angeboten. So kann man einen Film sehen und bekommt weitere Informationen auf dem Smartphone angezeigt. In manchen Spielen wird z.B. ein Smartphone zu einem zweiten interaktiven Zusatzbildschirm auf dem man weitere Informationen finden kann oder der weitere Aktivitäten im Spiel ermöglicht.

\section{XBOX Live}

XBOX Live ist ein Online-Netzwerk welches in einer kostenpflichtigen Gold-Version und einer kostenlosen Gratis-Version angeboten wird. Über die XBOX kann man sich in dem Netzwerk anmelden. Das Netzwerk bietet eine Vielzahl an Möglichkeiten, wovon ich im folgenden einige beschreiben möchte:

XBOX Live ist zugleich ein Marktplatz welcher den Zugang zu Spielen, Videos, Musik bietet und ein Netzwerk, mit dem sich die Spieler auf verschiedene Art und Weise vernetzen und miteinander kommunizieren können. So besteht die Möglichkeit miteinander zu chatten, zu reden (z. B. über ein Headset), Videochat über das KinectSystem sowie Nachrichten im Netzwerk zu versenden. 
Jeder Spieler benötigt einen eigenen Gamer-Tag über den z.B Spielstände abgespeichert werden oder aber neue gleichstarke Gegner in Multiplayer-Spielen zugewiesen werden können. Zudem besteht die Möglichkeit, innerhalb des Netzwerkes Nachrichten zu versenden.

Mit dem sogenannten „Gamertag“, einem individuellen Spielerprofil können innerhalb des Netzwerkes weitere Spieler gefunden werden, die ungefähr gleichstark sind, und gegen die man dann in bestimmten Spielen antreten kann.

Wenn man eine XBOX 360 einschaltet, landet man zuerst auf dem „Dashboard“. Dies ist quasi der Desktop der XBOX. Von hier aus kann man Spielen, Videos ansehen, ins Internet gehen, Kontakt mit anderen Spielern über XBOX Live aufnehmen etc. Die Funktionen werden immer wieder erweitert. Die Erweiterungen werden mit den jeweiligen Software-Updates eingespielt.

Die XBOX 360 wird im Moment durch die XBOX One ersetzt. Die XBOX ONE ist in allen Bereichen eine Weiterentwicklung und fokussiert sich noch mehr auf die Aufgabe als multioptionale Medienplattform. Das Kinect-System wird ebenfalls ausgebaut werden und ist elementarer Bestandteil der XBOX und kein optionales Add on.

Ebenfalls spannend sind neue Visualisierungsansätze der XBOX. Eine interessante Idee ist das Projekt „Illumi-Room“ welches Teil des Kinect-Systems werden könnte. Bei diesem Ansatz scannt eine Kamera den Raum in dem die Konsole steht und bindet dann durch Projektionen den Raum in das Spiel ein. Dieser Effekt ist auf den Videos des Microsoft Innovation Labors bereits zu sehen und erinnert ein bisschen an das Holo-Deck auf der Enterprise. Ob das System in der kommenden XBOX One bereits genutzt wird ist im Moment nicht absehbar. Es zeigt aber, das Räumlichkeit ein wesentliches Element zukünftiger Gaming-Konzepte sein wird.

Bei diesem System wird der Raum in dem man sich befindet in das Spiel integriert.

Die XBOX One ist nicht abwärtskompatibel! Das bedeutet, dass Sie auf der XBOX One keine Spiele der XBOX 360 spielen können. Sie müssen also einen komplett neuen Bestand aufbauen.

\section{Playstation 3}

\section{Anbieter: Sony}

Veröffentlichung in Europa: 23.03.2007

Anschlüsse: Strom, USB 2.0. HDMI, AV-Ausgang, Ethernet (LAN), Bluetooth, Optischer Digitalausgang

Sonstiges: WLAN, integrierte Festplatte

Die Playstation 3 ist eine stationäre Konsole des japanischen Unternehmens Sony. Sie verfügt über herausragende Grafikeigenschaften und ist ebenfalls in vielen Haushalten vorhanden. Die Konsole wurde Ende 2013 durch ihren Nachfolger die Playstation 4 abgelöst. Wie die XBOX 360 ist auch die Playstation 3 eine multioptionale Medienplattform. In diesem Fall ist die Basis das Playstation-Network. Jeder Spieler benötigt einen Account in diesem Netzwerk. Ohne einen Account bzw. eine ID kann die Playstation nur sehr eingeschränkt genutzt werden. Die ID ermöglicht wiederum das Spielen im Multiplayer-Modus. Das bedeutet, dass verschiedene Spieler vernetzt gegeneinander antreten. Diese Funktion ist kostenlos.

Im Gegensatz zur XBOX 360 kann die Playstation 3 auch Blue-Rays abspielen, was den Kauf eines weiteren Abspielgerätes unnötig macht. Ein besonderes Highlight 
für den Playstation-Spieler ist das Angebot „Playstation Plus“. Mit diesem Angebot entwickelt sich die Playstation in Teilen zu einem Flatrate-Dienst. Gegen eine Jahresgebühr von aktuell 49,99 € bekommt kann man z. B. Rabatte auf Spiele und kann zudem die am besten bewerteten Spiele kostenlos runterladen und spielen. Zudem gibt es einen exklusiven Zugriff auf bestimmte Inhalte wie z. B. Demos oder Trailer.

Als Medienplattform bietet die Konsole u. a. Zugang zu Sony-eigenem Filmen und Musik sowie weitere Unterhaltungsmedien via Lovefilm, Watchever, etc.

Im Laufe der Jahre wurde auch die Playstation 3 weiterentwickelt. Dabei wurden neuen Funktionalitäten hinzugefügt bzw. andere Optionen entfernt. Aktuell wird die Super-Slim-Version verkauft. Diese Version ist nicht abwärtskompatibel mit der Playstation 2 bzw. dem dafür produzierten Spielen.

Auch die Playstation 4 ist nicht abwärtskompatibel! Das bedeutet, dass Sie auf der Playstation 4 keine Spiele der Playstation 3 spielen können. Es soll ein paar Spiele der Playstation 3 geben, die online via Playstation 4 gespielt werden können. In diesem Fall fungiert die Playstation aber nur als Interface. Sie müssen also auch für diese Konsole einen komplett neuen Bestand aufbauen.

\section{Nintendo Wii und Wii U}

Die Konsolen des japanischen Herstellers Nintendo sind ebenfalls sehr erfolgreich. Im Gegensatz zu Sony und Microsoft hat Nintendo den Nachfolger der Wii die Wii U schon früher veröffentlicht. Da aber noch immer sehr viele Wii-Konsolen in den Haushalten stehen und genutzt werden, beschreibe ich hier beide Modelle.

\section{Nintendo Wii}

Anbieter: Nintendo

Veröffentlichung in Europa: 08.12.2006

Anschlüsse: Strom, USB 2.0, AV-Ausgang, Optischer Digitalausgang Sonstiges: WLAN

Die Nintendo Wii ist vor allem eine Familien-Konsole. Ihre Grafikleistung ist im Vergleich zur XBOX 360 oder der Playstation 3 eher schwach. Ihren Erfolg verdankt die Wii vor allem ihrem zum Zeitpunkt der Veröffentlichung revolutionären kabellosen Controllern. Diese Controller und die damit verbundenen Spiele, zielten vor allem auf Familien ab. Die angebotenen Spiele hatten zumeist einen sehr sozialen Charakter, d.h. man spielte zusammen mit Anderen.

Die Nintendo Wii ist nicht als multioptionale Medienplattform konzipiert worden. Zwar kann man mit ihr auch ins Internet gehen und es gab zu dem einige interessante multimediale Ansätze. Ihre Nutzungsmöglichkeiten sind aber vor allem im Vergleich mit der XBOX 360 und der Playstation 3 limitiert.

Wie die XBOX 360 und die Playstation 3 besitzt auch die Wii eine eigene Nutzeroberfläche. Hier werden die einzelnen Angebote in „Chanels“ eingeteilt. Ein großer Teil dieser Chanels wurde aber mittlerweile eingestellt. Interessant ist auch die „Virtual Console“ mit der man alte Spieler anderer Plattformen runterladen und spielen kann. Somit eignet sich die Konsole auch für das familiäre Retrogaming.

Ein Chanel erlaubt das Erstellen sogenannter „Miis“. Ein Mii ist ein individueller Avatar eines Spielers, wobei ein Spieler mehrere Miis erstellen kann. Ein Mii kann 
auch auf anderen Geräten von Nintendo wie z. B. auf dem Nintendo DS genutzt werden. Ein Mii bietet auch spezielle Vernetzungsmöglichkeiten kann.

Ein besonderes Highlight ist die Multiplayer-Option. Hierbei können sich Spieler überall auf der Welt online miteinander vernetzen und gegeneinander spielen. Diese Vernetzung ist auch auf anderen Nintendo-Geräten wie z. B. dem Nintendo DS möglich. In einigen Spielen kann man auch während des Spiels miteinander kommunizieren. Die Spieler können dafür aus einer vorgegebenen Anzahl von Nachrichten wählen. Diese werden dann an die Person gesendet, die man angesprochen hat. Da die Sätze vorgegeben sind, gibt es für jeden Satz Übersetzungen in vielen Sprachen. Daraus resultiert, dass z.B. jemand einen Satz auf Deutsch auswählt, dieser aber in Spanisch bei dem Adressaten ankommt, da dieser in Spanien lebt bzw. spanisch spricht.

\section{Nintendo Wii U}

Anbieter: Nintendo

Veröffentlichung in Europa: 30.11.2012

Anschlüsse: Strom, USB 2.0. HDMI, AV-Ausgang

Sonstiges: WLAN, kleine integrierte Festplatte

Die Nintendo Wii U ist der Nachfolger der Nintendo Wii. Das Besondere an diesem System ist der neuartige Controller, der an eine Mischung aus Gamepad und Tablet-PC erinnert. Das heißt, man findet sowohl klassische Steuerungselemente wie auch ein berührungsempfindliches Display vor. Es ist ein bisschen so, als würde man ein iPad in der Hand halten. Das bedeutet, man hat zwei Bildschirme zur Verfügung und die weitaus meisten Spiele nutzen diese Funktion intensiv.

Mit der Wii U wurde auch ein neues Online-Netzwerk gestartet. Mit „Miiverse“ gibt es nun eine Nintendo eigene Community. Sie greift auf das Nintendo-Netzwerk zurück. Der Zugang ist auch über einen PC oder ein Smartphone möglich. Die Spieler können nun nach gleichstarken Spielern suchen, mit anderen spielen, Screenshots erstellen und diese teilen, mit anderen kommunizieren etc.

Für das Jahr 2013 ist auch die Veröffentlichung bzw. die Integration von Nintendo Tvii angekündigt worden. Dabei handelt es sich um ein Angebot, welches den Zugang zu Videostreaming-Diensten wie z.B. Lovefilm ermöglichen soll. Das bedeutet, dass sich auch die Wii U hin zu einer multioptionalen Medienplattform entwickelt.

Zum Zeitpunkt der Erstellung dieses Buches bleibt die Wii U aber weit hinter den Erwartungen der Anbieter zurück. Die Verkaufszahlen sind schwächer als erwartet und die angebotenen Spiele sind nicht so interessant, dass sie die Konsole anschieben könnten. Insofern bleibt abzuwarten, ob die Wii U für Bibliotheken relevant ist.

Im Gegensatz zur Playstation und zur XBOX ist die Nintendo Wii U abwärtskompatibel. Das bedeutet, dass man auf dieser Konsole auch Spiele der Nintendo Wii spielen kann.

\section{Retro Konsolen}

Neben den hier genannten Konsolen gibt es natürlich noch viele weitere Konsolen. Neben absoluten Nischenmodellen und Prototypen sind es vor allem ältere Modelle, die für viele Gamer interessant sind. Zu diesen Konsolen gehören u. a. Nintendo NES, Nin- 
tendo 64, SEGA Megadrive, XBOX, Playstation 1 und 2, etc. Diese Systeme sind nicht nur für ältere Gamer interessant. Ich erlebe sehr oft, dass gerade jüngere Gamer sowohl die aktuellen als auch die veralteten Modelle schätzen und gerne mit Ihnen spielen. Es geht also offensichtlich nicht immer um eine High-End-Grafik und modernste Technologie.

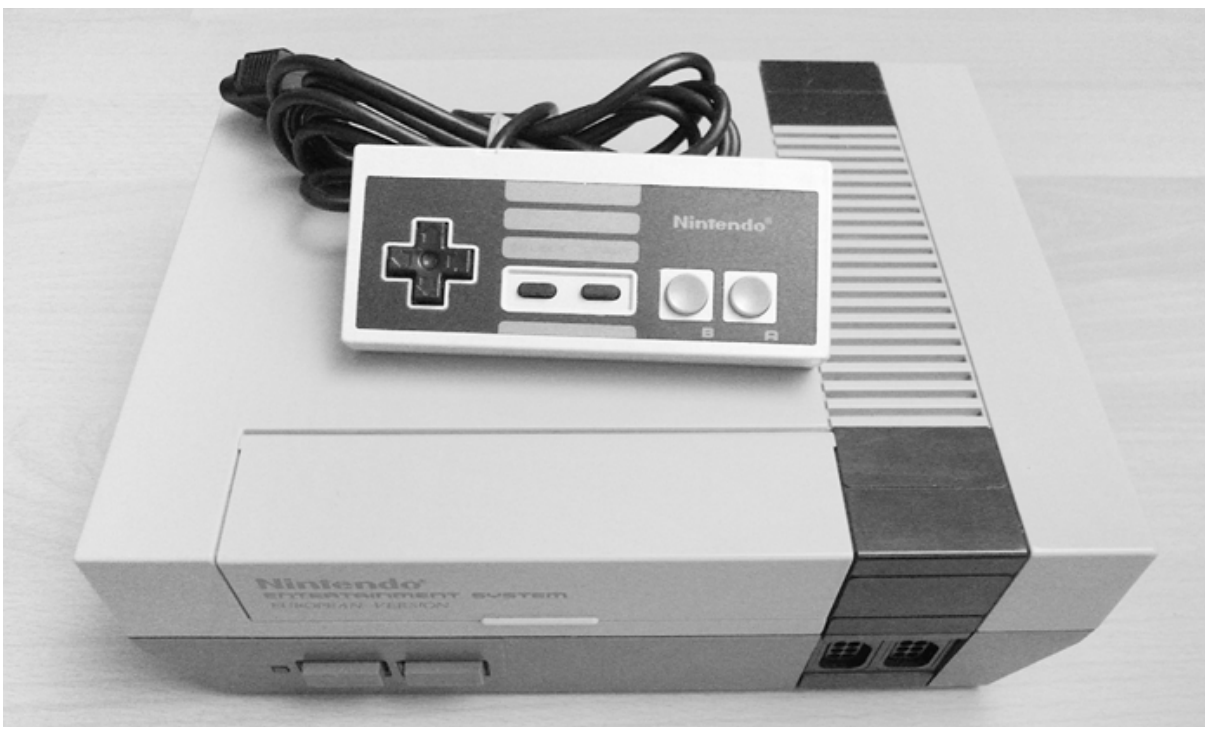

Abb. 6 Nintendo NES Konsole mit Controler.

Wenn Sie sich mit Retro-Konsolen beschäftigen möchten, gibt es ein paar wichtige Punkte zu beachten.

1. Retro-Konsolen sind in den meisten Fällen gebraucht. Das bedeutet, dass diese Systeme Kratzer etc. haben können.

2. Retro-Konsolen sind alt. Das bedeutet, dass sie irgendwann kaputt gehen werden.

3. Retro-Konsolen verfügen u.U. über andere Anschlüsse. Computer-Monitore mit HDMI, DVI oder VGA-Eingang werden dann nicht funktionieren.

4. Retro-Konsolen sind keine High-End-Konsolen. Damit Sie mich nicht falsch verstehen: Zum Zeitpunkt des Markteintritts waren diese Konsolen das Beste was man für Geld kaufen konnte. Aber heute sind diese Systeme veraltet. Erwarten Sie also nicht, dass die grafische Darstellung oder das Gameplay heutigen Standarts genügen.

Die Arbeit mit diesen Systemen ist grundsätzlich vergleichbar mit der Arbeit an aktuellen Systemen. Auch hier kann man sowohl die Hardware- als auch die Software erwerben. Allerdings muss man hierfür etwas intensiver recherchieren bzw. nach Anbietern suchen.

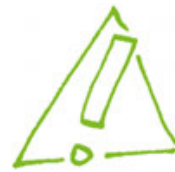

Fragen Sie im Umfeld Ihrer Bibliothek nach, ob jemand noch eine alte aber funktionierende Konsole nebst Spielen hat, die er Ihnen zur Verfügung stellen würde. Auf diese Art und Weise können sie Ihr Portfolio preiswert erweitern. Man kann dafür auch Patenschaften anbieten. In den meisten Fällen sind alte Konsolen noch funktionsfähig. Man muss sie nur aktivieren - und das ist 1000x spannender, als das System auf dem Dachboden zu lagern.

Es ist kein Problem, dass Retrokonsolen irgendwann kaputt gehen. Versuchen Sie nur unbedingt Ihre Kunden sehr früh zu informieren, dass die Systeme irgendwann ausfallen können. 


\section{Zusammenfassung und Nutzungsmöglichkeiten für Bibliotheken}

Wenn wir uns die aktuelle Konsolengeneration ansehen, so können wir feststellen, dass die mit den Systemen verbundenen Möglichkeiten stetig ausgebaut werden. Die Grafikleistung sowie die optionalen Nutzungsmöglichkeiten hinsichtlich dem Zugang $\mathrm{zu}$ anderen Medien sowie der Kommunikation der Spieler untereinander sind vielfältig. Durch die Möglichkeit, Inhalte runterzuladen bzw. zu streamen, werden diese Systeme zunehmend zu einer direkten Konkurrenz zu Bibliotheken, wenn es um die Auswahl und den Verleih von Medien wie z.B. Computerspielen, Filmen und Musik geht. Das Thema Bestandsarbeit werde ich noch in einem gesonderten Abschnitt besprechen. Zum jetzigen Zeitpunkt möchte ich nur dringend empfehlen, sich nicht auf Spiele für eine bestimmte Konsole zu beschränken. In manchen Fällen höre ich das Argument, dass man sich für Spiele für die Wii entschieden hat, weil man vor allem auf Familien eingehen möchte und diese Konsole vor allem bei Familien mit Kindern zu finden sei. Ich halte diesen Ansatz für nachvollziehbar aber auch für falsch. Die Playstation 3 und die XBOX 360 sind ebenso familienkompatibel. Zudem sollte bedacht werden, dass die Wii ausläuft und die Verkaufszahlen der Wii U nicht so sind, dass man zum jetzigen Zeitpunkt von einem ähnlichen Erfolg wie bei der Wii ausgehen kann.

Für Bibliotheken, die mehr machen möchten als nur Games zu verleihen, stellt sich die Frage, ob eine Anschaffung einer oder mehrerer Konsolen sinnvoll ist. Damit eng verbunden ist die Frage, welche Zielgruppe man mit den Gaming-Aktivitäten erreichen möchte. Alle beschriebenen Konsolen haben ihre Vor- und Nachteile. Bestimmte Funktionen wie das Streamen von Filmen und Musik werden in Bibliotheken keine große Rolle spielen. Auch wenn ich grundsätzlich der Meinung bin, dass sich alle Konsolen für die Nutzung in einer Bibliothek hervorragend eignen, ist die XBOX 360 und ihr anstehender Nachfolger die XBOX One durch ihr Kinect-System besonders interessant für den Einsatz in Bibliotheken. Der Grund ist der, dass der Spieler keinen Controller mehr braucht, da sein eigener Körper als Controller genutzt wird. Es gibt also keine bewegliche Hardware auf die man achten muss bzw. die kaputt gehen könnte. Die Playstation 4 und die Playstation 3 haben wiederum den Vorteil, dass Sie in den meisten Fällen die beste Grafik bieten und dass sehr viele Gamer diese Konsole bevorzugen. Die Wii U kann hier kaum Punkten. Das neue Controllerkonzept ist nur in Teilen wirklich spannend und wird auf den Konkurrenzkonsolen durch die Einbindung von iPad und Co. ebenfalls ermöglicht. Allenfalls die Abwärtskompatibilität ist ein Argument, welches für die Wii U spricht. Wobei man beachten sollte, dass es es auch in den nächsten Jahren neue Spiele für die XBOX360 und die Playstation 3 geben wird.

Abraten möchte ich vom Verleih von jeglicher Hardware. Die heutigen Systeme sind immer an einen Spieler gebunden. Zwar kann man diese Bindung jederzeit wieder aufheben, Jedoch ist dies mit einem erheblichen Aufwand verbunden. Wenn eine Bindung an die Konsole aber nicht möglich ist, können die Spieler ihre Erfolge nicht speichern und mitnehmen. Das im Bereich eBooks desöfteren angewandte Modell, eBook-Reader zu verleihen, um den Bibliothekskunden die Möglichkeit zu geben, die Reader auszuprobieren, ist in diesem Bereich nicht notwendig. Möchten Sie also Konsolen erwerben, dann geht es dabei um die Nutzung in der Bibliothek, sei es als dauerhaftes oder als temporäres Angebot.

Auch wenn Sie für Ihre Bibliothek keine Konsole anschaffen möchten, müssen Sie sich trotzdem damit beschäftigen. Sie müssen dabei nicht alle Funktionen bis ins Detail beherrschen. Folgende Kenntnisse sind von Bedeutung: 

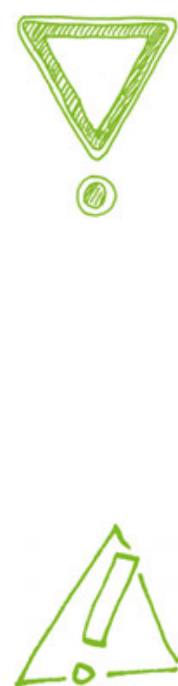

1. Wie baue ich eine Konsole auf und wie schließe ich sie an die Peripherie (Monitor, Lautsprecher etc.) an

2. Wie aktiviere ich die Controller?

3. Wie lade ich die Controller auf?

4. Wie starte ich ein Spiel?

5. Wie nutze ich die Controller?

6. Wie funktionieren die weitergehenden Angebote der jeweiligen Plattformen?

7. Wie führe ich Systemupdates durch?

8. Wie aktiviere ich den Internetzugang?

9. Wie erstelle ich ein Profil?

10. Wie funktionieren die jeweiligen Communities bzw. Online-Netzwerke?

Suchen Sie auf Youtube nach Tutorial-Videos zu den genannten Punkten. Sie können auch Gamer fragen, ob diese Ihnen helfen können.

Im Gegensatz zur Wii U sind die XBOX One und die Playstation 4 nicht abwärtskompatibel. D.h auf diesen Konsolen lassen sich keine Spiele der Vorgängerkonsolen spielen. Auf der Wii U ist dies aber möglich. Es kann sein, dass es über Cloud-Gaming möglich ist, Spiele für Vorgängerkonsolen zu spielen. Dabei wird das Spiel auf einem externen Server gespielt und dann per Videostream mit der Konsole gespielt. Dabei wird die Konsole also „nur“ als Eingabegerät genutzt.

\section{Controller}

Nachdem Sie die Konsolen kennengelernt haben, ist es nun wichtig, dass wir uns mit den Controllern beschäftigen. Ich habe lange darüber nachgedacht, ob ich die Controller bei den jeweiligen Vorstellungen der Konsolen beschreiben sollte. Ich bin aber zu der Überzeugung gelangt, dass es besser ist, dem Thema Controller einen eigenen Abschnitt zuzuweisen. Der Grund dafür ist folgender: Es gibt eine Vielzahl an Controllern. Diese werden nicht nur von den Herstellern der Konsolen produziert. Zudem gibt es für viele Spiele Spezialanfertigungen. Für Bibliotheken ist aber sehr wichtig, auch in diesem Bereich über Wissen zu verfügen. Zum Einen können Sie erst dann eine sinnvolle Beratung zum Thema anbieten, und zum Anderen ist dieses Wissen für ihre eigenen Aktivitäten in diesem Bereich relevant.

Beginnen wir also mit etwas Basiswissen. Schon bei den ersten stationären Konsolen gab es Controller. Mit ihnen wurde und wird eine Spielfigur oder ein Gegenstand gesteuert. Der Controller bildet quasi das Interface zwischen Spieler und Spiel. Das bedeutet, dass der Spieler nicht nur das Spiel sondern auch den Controller bzw. seine Funktionen verstehen bzw. erlernen muss. Je komplexer ein Controller aufgebaut ist, desto mehr Funktionen habe ich als Spieler zur Verfügung. Ich muss aber alle diese Funktionen zuerst erlernen.

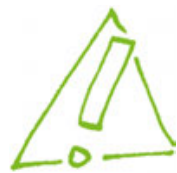

Wenn Sie in ihrer Bibliothek einen Gaming-Event durchführen möchten und dazu jeden Menschen einladen, der mitmachen möchte (egal ob Anfänger oder Fortgeschrittener) sollten Sie für die Veranstaltung Spiele auswählen, bei denen sowohl das Spiel als auch die Funktionsweisen des Controllers einfach und schnell zu erlernen sind. Um auszuprobieren, ob sich das Spiel für eine derartige Veranstaltung eignet, bitten Sie am besten einen Kollegen mit geringer bis gar keiner Gaming-Erfahrung, das jeweilige Spiel zu spielen. Wen diese Person das Spiel spielen kann, sollte es auch bei den meisten anderen Teilnehmern funktionieren. 
Die ersten Controller waren verhältnismäßig einfach. Es gab nur wenige Nutzungsmöglichkeiten. Die vielleicht einfachste Variante war der Joystick. Dieser Controller ähnelte stark einem Steuerknüppel eines Flugzeuges. Er konnte in verschiedene Richtungen bewegt werden. In der einfachen Version war zudem noch ein Feuerknopf vorhanden. Mit zunehmender Komplexität der Spiele wurden auch die Controller immer komplexer. Heutige Controller haben eine große Zahl an verschiedenen Tasten. Jede dieser Tasten hat eine eigene Funktion. Zudem gibt es spezielle Tastenkombinationen. In einigen Spielen sind die Tastenbelegung und die Tastenkombinationen frei programmierbar.

Die Entwicklung der Controller geht aber noch weiter. Heutige Controller sind zumeist kabellos und haben eine Vibrationsfunktion. Letzteres bedeutet, dass der Spieler ein direktes haptisches Feedback auf das Spielgeschehen bekommt.

\section{Nintendo Wiimote}

Die Controller der Nintendo Wii, die sogenannte „Wiimote“, geben ihre Signale nicht nur mittels der Tasten an die Konsole weiter. Die Wiimote ist mit einem Bewegungssensor sowie einer Infraroterkennung mittels Sensorleiste ausgestattet. Diese Konfiguration sorgt dafür, dass man durch eine Kombination aus Bewegungen des Controllers und dem Drücken von Tasten des Controllers eine neue Form der Steuerung von Computerspielen hat. Konkret kann man mit diesem Controller beispielsweise Tennis spielen und dabei den Controller wie einen Tennisschläger bewegen. Mit diesen Funktionen ergab sich ein völlig neues Spielgefühl. Erweitert wurde die Wiimote durch den „Nunchuck“. Zudem gab es für die Wiimote Erweiterungen, die keine eigene Funktion hatten. So gab es ein Lenkrad oder einen Tennisschläger, in die die Wiimote eingefügt wurde. Somit veränderte sich das Spielgefühl nachhaltig, weil man nun einen ganzen Tennisschläger in der Hand hielt. Die Wiimote ist zudem mit einem Lautsprecher sowie ebenfalls einem Vibrationssystem ausgestattet.

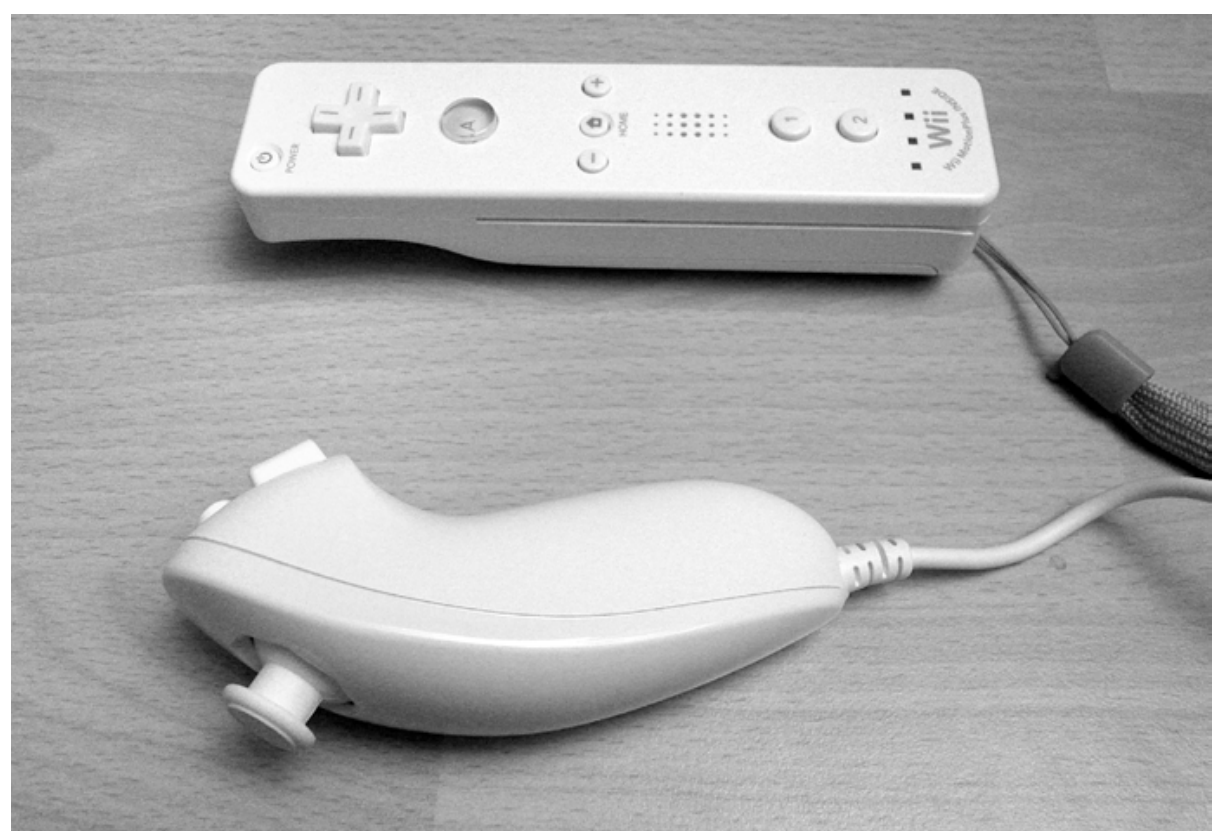

Abb. 7 WiiMote und Nunchuck. 


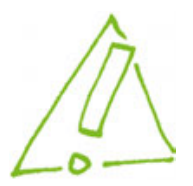

Achten Sie beim Kauf einer Wii oder beim Kauf von einzelnen Controllern, das diese mit „MotionPlus“ ausgestattet sind. Diese Erweiterung verfeinert die Erfassung der Bewegungen der Wiimote. Sollten Sie noch alte Controller ohne MotionPlus haben, rate ich dringend, die Erweiterung zuzukaufen.

\section{Nintendo Wii U-Controller}

Dieser Controller ist quasi eine Mischung aus einem Tablet wie z. B. dem iPad und einem Controller. Es verfügt ebenso wie die Wiimote über eine Bewegungssteuerung. Es sind natürlich einige klassische Controller-Tasten vorhanden. Zentrales Element ist aber der 6,2" große Touchscreen. Er hat verschiedene Funktionen. Zum Einen ist er ein weiterer Bildschirm, auf dem in Spielen weitere Informationen eingeblendet werden können. Zum Anderen kann man auch alleine mit dem Controller spielen - wenn etwas der Fernseher gerade besetzt ist. Der Controller hat zudem ein Mikrofon und Lautsprecher, eine Kamera für Videochat und einen NFC-Kontaktpunkt der es dem System ermöglicht, Spielkarten auszulesen und deren Inhalte in das Spiel zu integrieren. Eine TV-Taste ermöglicht zudem, das Gamepad als Fernbedienung für den TV zu nutzen.

Die Nutzungsmöglichkeiten für diesen Controller sind vielfältig. Gute Spieleentwickler werden in Zukunft versuchen, alle daraus resultierenden Optionen und Anwendungen in die Spiele zu integrieren.

\section{Controllerversionen am Beispiel der Playstation 3}

Das Universum der Controller ist riesig. Es gibt eine große Zahl an Spezialanfertigungen, welche nur für bestimmte Spiele entwickelt wurden. Am Beispiel der Playstation 3 möchte ich aufzeigen, welche verschiedenen Controller es gibt. Wie bereits erwähnt, wird die Playstation 3 mit einem Standart-Controller ausgeliefert. Dieser Controller ist kabellos und verfügt über die bereits angesprochene Feedback-Funktion.

\section{Playstation Move}

Eine weitere Variante ist Playstation Move. Dieser Controller ähnelt der Idee hinter der Wiimote und ist ebenfalls ein bewegungsempfindlicher Controller.

\section{Wireless Buzz}

Hierbei handelt es ich um einen Controller der dem Buzzer in einer Quizsendung nachempfunden ist. Er wurde ursprünglich für das Quizspiel „Buzz!“ entwickelt. Wenn der Quizmaster eine Frage stellt kann man den Buzzer drücken und sich danach durch drücken einer der farbigen Knöpfe für eine Antwort entscheiden.

\section{Playstation Guitar}

Auf der Playstation kann man Musik-Spiele spielen. Ein sehr erfolgreiches Spiel ist „Guitar-Hero“ Dabei spielt man berühmte Rock-Songs nach. Für dieses Spiel wurde 


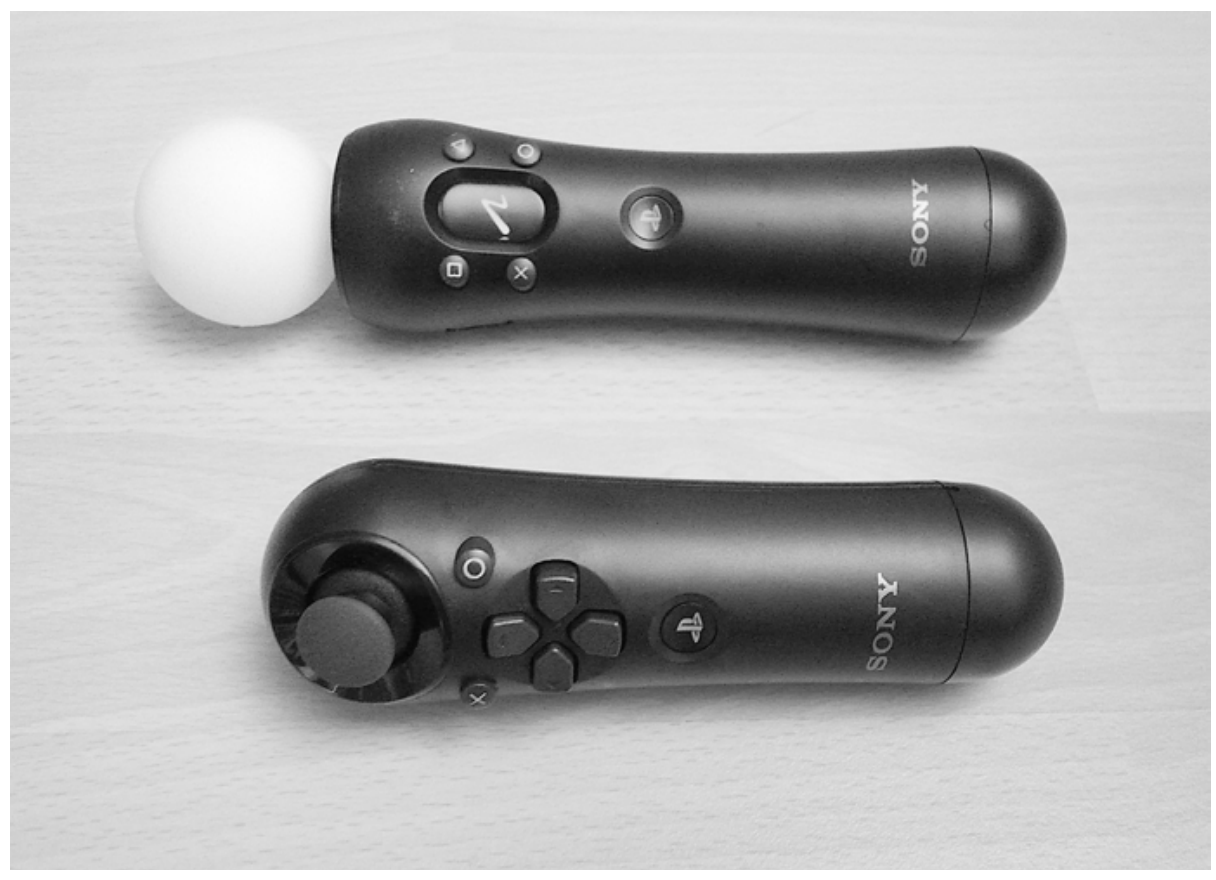

Abb. 8 Playstation Move Controller.

ein Controller in Form einer Gitarre entwickelt. Der Controller hat fünf farbige Tasten. Das drücken dieser Tasten legt die Tonhöhe fest. Zudem ist eine Art Kippschalter vorhanden. Er liegt an der Stelle, an der man bei einer echten Gitarre die Saiten anschlagen würde. Die Aufgabe des Spielers ist nun, die richtigen farbigen Tasten und den Kippschalter im Takt zu betätigen.

\section{Skateboard-Controller für „Tony Hawk Ride“}

Für das Spiel „Tony Hawk Ride“ wurde ein sogenannter Skateboard-Controller entwickelt. (Das Spiel und den Controller gibt es auch für die Nintendo Wii und die XBOX 360). Der Controller hat die Form eines Skateboards. Er ist mit Bewegungssensoren ausgestattet. Auf diese Art und Weise steht man auf dem Board und kontrolliert die Spielfigur durch die eigenen Körperbewegungen.

\section{Kinect - eine Revolution in der Welt der Controller}

Ein Element der XBOX 360 bzw. der XBOX One ist besonders interessant: das KinectSystem. Hierbei handelt es sich um eine völlig neue Art eines Controllers. Bei allen anderen Konsolen haben Sie einen physischen Controller in der Hand. Das Aussehen und die Nutzung der Controller mögen variieren, es bleibt aber dabei, dass sie etwas in der Hand halten. Das Kinect-System macht Ihren ganzen Körper zum Controller - ohne dass Sie selber etwas in der Hand halten müssen. Das Prinzip ist denkbar einfach. Kinect besteht aus einer kleinen Leiste in die ein Kamerasystem sowie Mikrofone eingebaut sind. Diese Leiste wird vor dem Spieler positioniert. Die Kamera scannt die Bewegungen des Spielers und die können so in die jeweiligen Spiele übernommen werden. 


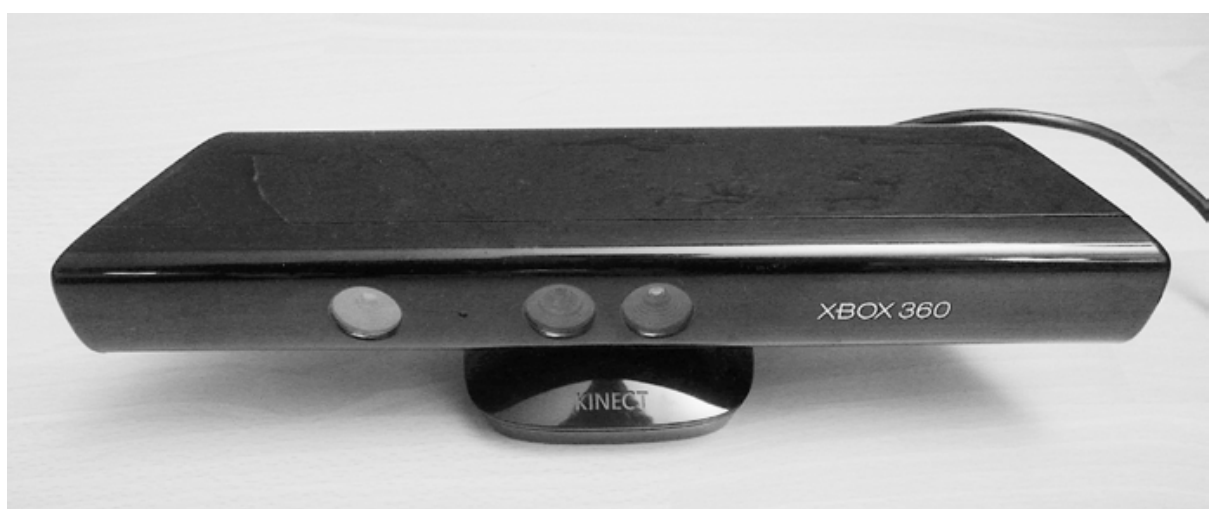

Abb. 9 Kinectsystem für XBOX360.

Die Nutzungsmöglichkeiten sind vielfältig. In Autorennen lenken Sie aktiv mit Ihren Händen bzw. Ihrem ganzen Körper den Rennwagen. In Science-Fiction-Abenteuern erleben Sie Kämpfe mit Lichtschwertern durch Bewegung Ihrer Arme. In anderen Spielen streicheln Sie Babys von Raubkatzen und erleben mit ihnen verschiedene kleine Abenteuer etc.

Durch die Einbindung des menschlichen Körpers werden die Spiele so zu „Bewegungsspielen“ und man kommt dabei sehr oft ins Schwitzen. Und natürlich kann man manchen Spielen auch Tanzen lernen oder sich mit Aerobic fit halten.

Das Kinectsystem verfügt zudem über Mikrofone wodurch auch die Sprache des Spielers Teil des Spieles wird. So kann man in Kinectimals seinem Raubtierbaby einen Namen geben und es dann rufen. Besonders spannend sind die Nutzungsmöglichkeiten im Spiel FIFA. Bei diesem Spiel steuern Sie mittels klassischem Controller eine Fussballmanschaft. Wenn Sie nun über bestimmte Schiedsrichterentscheidungen so erbost sind, dass Sie laut fluchen und sich aufregen, dann hat dies einen realen Einfluss auf das Spiel. Sie werden u.U. aufgrund ihrer Beschwerde verwarnt.

Kinect erlaubt eine völlig neue Form des Gamings. Der eigene Körper als Controller bedeutet eine andere Wahrnehmung des Spiels und auch eine andere Reaktion des Körpers, des Raumes bzw. des Spielers auf das Spiel.

Das Kinect-System findet auch Anwendung in der Funktion der XBOX 360 als Medienzentrale eines Haushalts. Die Gestensteuerung wird auch benutzt um z. B. Videos oder Musik auszusuchen. Mit Kinect ist das Thema Gestensteuerung in die Haushalte gekommen. Nur ein paar Jahre nach dem Verkaufsbeginn von Kinect ist die Gestensteuerung zu einem Standard in modernen Haushalten geworden. Immer mehr Geräte, allen voran die überaus erfolgreichen Smart-TVs verfügen ebenfalls über eine eingebaute Gestensteuerung und auch in aktuellen Smartphones kommt dieses Modell zum Einsatz.

\section{Zusammenfassung und Empfehlungen für Bibliotheken}

Es gibt eine Vielzahl an unterschiedlichen Controllern. Mit der Weiterentwicklung der Konsolen wurden auch deren Controller immer komplexer bzw. an die jeweilige Funktion bzw. das jeweilige Spiel angepasst. Microsofts Kinect ist insofern eine Besonderheit, als dass es hier keinen physischen Controller mehr gibt. Der Körper des Spielers wird zum Controller und der reale Raum wird Teil des Spiels. Aber auch die anderen Angebote sind spannend. Die Bewegungssteuerung in den Move-Controllern für die Playstation und der Wiimote für die Wii bzw. die Wii U ermöglichen eine Vielzahl an 
spannenden Spielen. Gerade die Interaktion mit dem Spiel und des eigenen Körpers erzeugt auch eine neue Form des Zuschauens. Nicht mehr nur das Geschehen auf dem Bildschirm, sondern ebenfalls die Bewegungen der Spieler sind interessant.

Die Erweiterungen wie z.B. die Gitarre für Guitar-Hero oder aber das Skateboard für Tony Hawk erzeugen beim Spieler ein Gefühl von Authentizität, auch wenn das Spiel wenig mit dem Spiel auf einer echten Gitarre bzw. dem Fahren eines echten Skateboards zu tun hat.

Für Bibliotheken ist es nun wichtig, sich einen Überblick über die verschiedenen Angebote zu verschaffen. Dabei sollten Sie wenn möglich die einzelnen Angebote selber ausprobieren. Erst dann können Sie abschätzen, wie lange es dauert, bis man den Controller beherrscht. Sie sollten auch wissen, welche Spiele einen speziellen Controller benötigen. Dies ist zum einen relevant bei der Information zu spielen, welche im Bestand einer Bibliothek zu finden sind. Zum anderen sollten Sie abschätzen können, ob Sie bestimmte Controller in der Bibliothek bei einem stationären Angebot vorhalten sollten. Controller sind also mehr als etwas komplexere Eingabegeräte. Und sie sollten gut funktionieren. Aus diesem Grund rate ich auch dringend dazu, möglichst Original-Hardware zu kaufen. Es gibt für sehr viele Anwendungen auch preiswerte Nachbauten. Meine Erfahrung hat aber gezeigt, dass die Lebensdauer mancher Nachbauten viel geringer als beim Original ist. Gerade für Bibliotheken, die z. B. GamingEvents anbieten möchten, ist es notwendig, Hardware zu kaufen, die lange hält.

\section{Peripherie}

Neben den beschriebenen Konsolen werden verschiedene Peripherie-Geräte benötigt. Einige der Geräte sind grundsätzlich notwendig um überhaupt spielen zu können, andere Geräte sind nicht zwingend notwendig, verbessern aber das Spielerlebnis. Beginnen wir zuerst mit den Geräten die Sie unbedingt benötigen, wenn Sie mit stationären Konsolen arbeiten möchten:

\section{Monitore}

Es gibt natürlich eine Vielzahl Monitoren auf dem Markt und letztlich müssen Sie selbst entscheiden, welchen Monitor Sie benötigen. Ich möchte aber ein paar Empfehlungen aussprechen. Die Bildschirmdiagonale sollte mindestens 24“ betragen. Die Reaktionszeit sollte, wenn möglich, $2 \mathrm{~ms}$ betragen. Der Monitor sollte über folgende Anschlüsse verfügen: HDMI, DVI, VGA und Audio-out (3,5 mm Stereo-Klinke). Wenn Sie die Konsole mittels einem HDMI-Kabel an den Monitor anschließen, wird nicht nur das Bild, sondern auch der Ton übertragen. Dieses Audio-Signal können Sie dann mit einem Stereo-Klinkenkabel an einem Lautsprecher übertragen. Wenn möglich, sollte auch das Kontrastverhältnis möglichst hoch sein.

\section{Lautsprecher}

Manche Monitore verfügen auch über eigene Lautsprecher. In den meisten Fällen ist dies aber nicht der Fall. Zudem sind diese Lautsprecher in der Regel klanglich nicht $\mathrm{zu}$ empfehlen. Aus diesem Grund empfehle ich dringend eigene Lautsprecher zu besorgen. Für ca. 40,00 € bekommen Sie sogenannte 2.1 Systeme, was bedeutet, dass Sie einen Subwoofer und zwei Zwei-Wege-Satelliten erwerben. 


\section{Batterien/Akkus nebst Ladegeräten}

Vor allem die Controller müssen kontinuierlich aufgeladen werden. Bei Controllern für die Playstation können Sie die Akkus nicht austauschen. Zum Aufladen müssen Sie den Controller an die Playstation anschließen, damit er wieder auflädt. Bei den anderen Controllern können Sie die Akkus austauschen. Ich empfehle unbedingt keine Einweg-Batterien sondern unbedingt wieder aufladbare Akkus zu verwenden. Das Ladegerät sollte kein Einsteigermodell sein. Investieren Sie lieber ein paar Euro mehr und kaufen Sie ein Ladegerät welches die Akkus schont und vollständige Entladungen ermöglicht. Jeder einzelne Schacht sollte überwacht und gesteuert werden können.

\section{Internetzugang}

Wie bereits erwähnt ist ein Internetzugang für alle beschrieben aktuellen stationären Konsolen notwendig. Nur so können Sie Systemupdates herunterladen. Diese Updates beinhalten sehr oft auch neue Funktionen die Sie für bestimmte Spiele brauchen. Der Internetzugang kann auch über WLAN erfolgen, sollte aber in diesem Fall schnell genug sein.

Darüber hinaus gibt es Zubehör, welches nur bei bestimmten Spielen relevant ist:

\section{Mikrofone}

Für einige Spiele wie z.B. SingStar gibt es spezielle Mikrofone. Diese Spiele ähneln Karaoke-Programmen. Diese Mikrofone sind nicht besonders teuer - Spiele wie SingStar sind aber noch immer interessant für die Nutzung bei Gaming-Events.

\section{Kameras}

Für einige Spiele sowie für Videochats und ähnliche Anwendungen werden Kameras benötigt. Dabei muss man unterscheiden zwischen Kameras, die zur Konsole gehören und solchen, die z.B. an einen Computer angeschlossen werden. Letzte sind sogenannte Webcams. Webcams sollten eine hohe Auflösung haben. Ob Sie wirklich eine Kamera passend zur Konsole brauchen, ist abhängig von den Spielen, die Sie spielen möchten. In einigen Fällen wird bei einem Spiel, welches eine solche Kamera benötigt, die Kamera mitgeliefert. Vor allem bei den immer erfolgreicher werdenden Augmented Reality-Games sind Kameras notwendig.

\section{Headsets}

Vor allem wenn man Spiele im Multiplayermodus spielt, sind Headsets eine sinnvolle Investition. Die Kommunikation mit den anderen Spielern wird so massiv erleichtert. Bei einigen Spielen können Sie zudem Sprachbefehle eingeben, um z. B. Maschinen $\mathrm{zu}$ aktivieren. Wenn es nur um die Kommunikation mit anderen Spielern geht, kann man auch auf Skype oder Teamspeak zurückgreifen. 


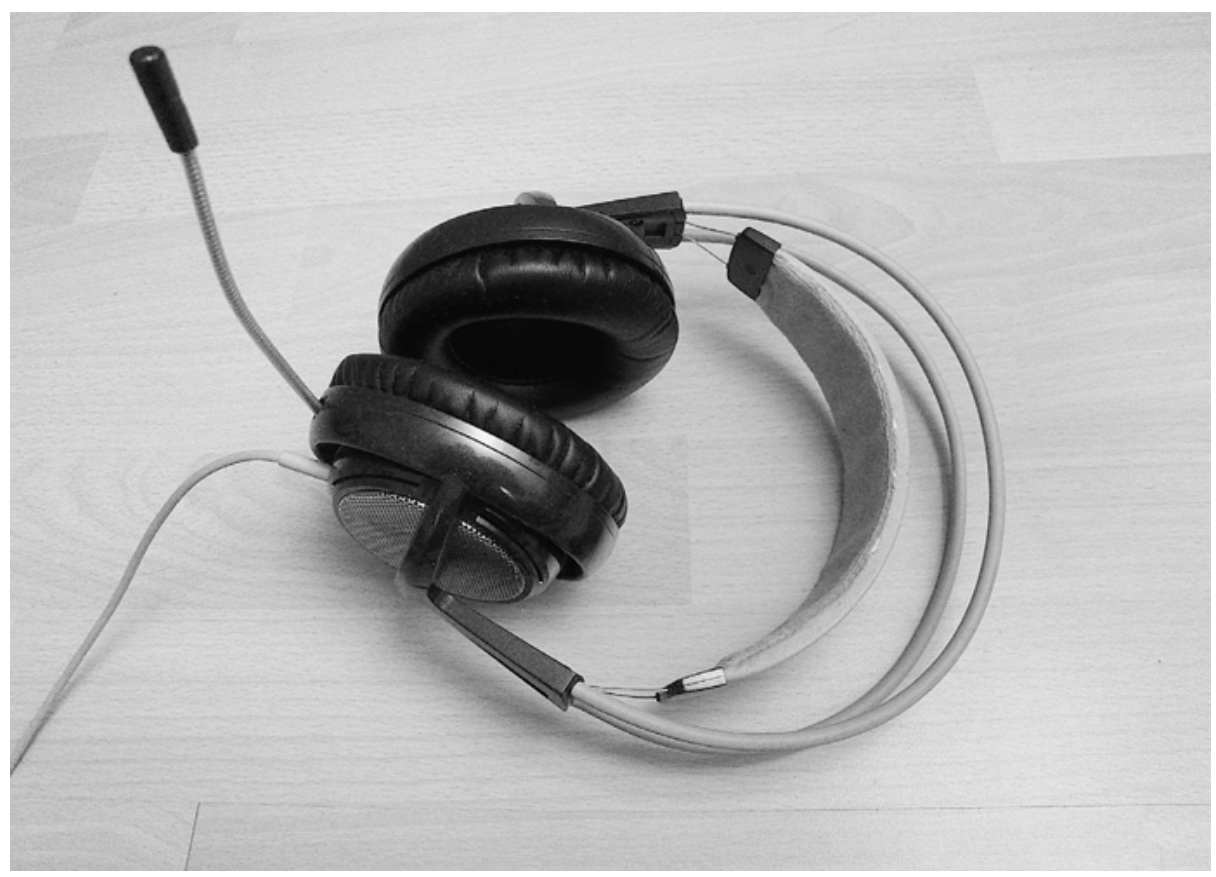

Abb. 10 Beispiel für ein professionelles Gaming-Headset.

\section{Mobile Konsolen}

Neben den stationären Konsolen, sowie den Heimcomputern sind vor allem die mobilen Konsolen relevant. Ich kenne beispielsweise nur wenige Menschen, die nichts mit dem Wort „Gameboy“ anfangen können. Die mobilen Geräte ermöglichen es, quasi überall zu spielen. Meiner persönlichen Meinung nach sind mobile Konsolen aber mehr als nur ein kleines Spielzeug. Ich kann mir nicht vorstellen, dass es ohne die mobilen Gaming-Konsolen zu einem derartigen Erfolg der Smartphones gekommen wäre. Die mobilen Konsolen „trainierten“ eine ganze Generation im Umgang mit mobilen Geräten, die wiederum immer komplexer wurden.

Mobile Konsolen sind letztlich eine Verschmelzung zwischen Controller, Konsole und Monitor. Damit ist es möglich, überall zu spielen.

Die Geschichte der mobilen Konsolen (oder auch Handheld-Konsolen genannt) beginnt schon in den 70ern des vorigen Jahrhunderts. 1979 kam die erste Konsole mit auswechselbaren Modulen auf den Markt. Ihr Name war „Microvision“. Leider war das System kein kommerzieler Erfolg. Erst mit dem Aufkommen des „Gameboy“ von Nintendo wurden die mobilen Konsolen zu einem wirklichen Massenphänomen. 1990 wurde in Europa der GameBoy veröffentlicht. Nintendo hatte davor u. a. mit dem ebenfalls mobilen Spielsystem „Game \& Watch“ große Erfolge gefeiert. Der Unterschied war dabei der, dass die Spielmodule im Gameboy austauschbar waren. Der Gameboy war ein riesiger Erfolg. Er ging einher mit weiteren Experimenten wie z. B. den Tamagotschis, welche in Deutschland 1997 auf den Markt kamen. Auch heute steht eine Vielzahl an mobilen Angeboten zur Verfügung. Der Nintendo DS in seinen verschiedenen Ausführungen, die „Playstation Portable“ und ihr Nachfolger die „Playstation Vita“ und natürlich die Welt der Smartphones und Tablet-PCs haben Spielen mobil gemacht.

Die ersten Konsolen waren reine Gaming-Konsolen. Erst später wurden die Funktionen erweitert. So kann man heute mit Ihnen ins Internet gehen, skypen, chatten, vernetzt spielen etc. Es gibt eine Vielzahl an Möglichkeiten und letztlich haben sich 
auch die mobilen Konsolen zu multioptionalen Medien- und Kommunikationsplattformen entwickelt. Inwieweit diese Systeme eine Zukunft haben bleibt abzuwarten. Gerade die Smartphones sind zu ernsthaften Konkurrenten geworden. Auf Smartphones und Tablet-PCs gehe ich noch gesondert ein. Im Folgenden möchte ich kurz die relevanten mobilen Konsolen beschreiben. Auch hier geht es nicht um eine komplette Abhandlung aller Möglichkeiten und Spezifika. Mir geht es vor allem darum, dass Sie verstehen, was diese Systeme können und in welcher Form sie aus Sicht von Bibliotheken nutzbar sind.

\section{Nintendo Gameboy}

Der Nintendo Gameboy ist sicherlich neben dem Nintendo DS die bekannteste mobile Gaming-Konsole. Das Display war verhältnismäßig schwach. Bis zur Einführung des Gameboy Colour 1998 handelte es sich zudem um ein Monochrom-Display. Erst der Gameboy Colour hatte ein farbiges Display. Das Display war nicht berührungsempfindlich. Der Gameboy war multiplayerfähig. Hierfür benötigte man eine stationäre Konsole, den Nintendo Game-Cube. Diese wurde zur Schnittstelle zwischen den einzelnen Systemen. Die Vernetzung wurde mittels spezieller Kabel (später auch WIFI) und einer Vernetzungssoftware ermöglicht. Die Möglichkeit, die Spielmodule auszutauschen sorgte dafür, dass immer mehr Spiele für den Gameboy entwickelt und verkauft wurden.

\section{Nintendo DS}

Der Nintendo DS ist nicht der Nachfolger des Gameboys. Er ist vielmehr ein System, welches parallel zum Gameboy entwickelt und verkauft wurde. Bis heute wird der Nintendo DS, wenn auch in abgewandelter Form produziert und verkauft. Der wesentliche Unterschied zwischen dem Gameboy und dem Nintendo DS sind die beiden Bildschirme des aufklappbaren DS, wobei der untere Bildschirm berührungsempfindlich ist. Daraus resultiert ein völlig neues Gaming-Gefühl. Das Spiel wird sowohl mit den Tasten als auch mit dem berühungsempfindlichen Display gespielt. Eine weitere Steuerung ist in manchen Spielen über das eingebaute Mikrofon möglich. Mit dem Nintendo DS ist es auch möglich, vernetzt mit anderen Spielern zu spielen. Hierfür wird entweder ein lokales Netzwerk geschaffen, in dem man die Konsolen kabellos miteinander verbindet. Oder aber man verbindet die Konsole über WLAN mit dem Internet. Ein spezieller Dienst von Nintendo mit dem Namen „Nintendo WIFI Connection“ ermöglicht nun das gegeneinander spielen auch über große Entfernungen. Ebenso möglich war die Vernetzung mit einer Wii.

Der ursprüngliche Nintendo DS war abwärtskompatibel, d.h. man konnte auf dem System auch Spiele für den Gameboy spielen. Die Kompatibilität besteht ab dem Nintendo 3DS jedoch nicht mehr.

Der Nintendo DS wird aber nicht nur zum Spielen genutzt. Es gibt eine Vielzahl an weiteren Funktionen. Man kann mit dem Nintendo DS auch im Internet surfen oder mit anderen chatten. Ebenso gibt es eBooks für den Nintendo DS.

Im Jahr 2006 erschien der Nintendo DS Lite. Das System ist kleiner und handlicher und verfügt über bessere Displays, bessere Lausprecher, eine bessere Verarbeitung ein moderneres Design etc.

2009 erschien dann das Nintendo DSi. Hier ist der Schacht für die GameboySpiele verschwunden. Das System verfügt über zwei VGA-Kameras sowie eine Slot für 
SD-Cards. Die Kameras ermöglichen das Aufnehmen von Bildern, welche u. a. direkt auf Facebook hochgeladen werden können. Der OPERA-Browser für den Zugang ins Internet ist nun kostenlos verfügbar.

2010 erschien der Nintendo DSi XL. Dieses Modell ist um einiges größer als der normale Nintendo DSi.

\section{Nintendo 3DS}

Am 25.03.2011 kam dann der Nintendo 3DS auf den Markt. Er löste den Nintendo DS ab. Das Besondere an dieser Konsole ist die Darstellung von Spielen in 3D ohne dass dafür eine spezielle Brille notwendig ist. Der 3DS verfügt über eine Bewegungssteuerung bzw. Bewegungssensoren, die zum Einen in Spielen und zum Anderen in weiteren Applikationen angewendet werden. Dazu gehört zum Beispiel ein Schrittzähler, der die zurückgelegten Schritte des Spielers zählt und den Spieler für je 100 Schritte mit einer Spielmünze belohnt. Dies bedeutet eine Motivation, sich zu bewegen. Es bedeutet aber auch, dass das System ständig mitgeführt werden muss. Der 3DS verfügt auch über ein Kamerasystem, welches Aufnahmen in 2D und 3D ermöglicht. Auf dem 3DS können auch Spiele des Nintendo DS abgespielt werden.

Besonders interessant sind die Community-Funktionen und hier insbesondere der sogenannte Street-Pass. Hierbei handelt es sich um eine Funktion, die es dem Spieler ermöglicht, kabellos Informationen wie z. B. Mii-Daten oder Spielstände auszutauschen. Dieser Austausch geschieht auf Wunsch automatisch. Das bedeutet, das Gerät verbindet sich automatisch mit einem anderen mit einem anderen $u$. $U$. fremden Gerät, wenn es sich in der Nähe befindet. Dabei werden keine personenbezogenen Daten ausgetauscht. Der Austausch bezieht sich nur auf die Mii's und die Spieldaten.

\section{Playstation Portable}

Neben Nintendo ist auch der japanische Konzern Sony im Bereich der mobilen Konsolen aktiv. Im Jahr 2005 kam die Playstation Portable (kurz: PSP) auf den Markt. Die PSP hatte ein sogenanntes UMD-Laufwerk, auf dem die Spiele und weitere Medien wie z.B. Filme konsumiert werden konnten. Dieses Laufwerk verschwand im Laufe der Jahre durch die Entwicklung neuer Versionen der PSP. Die Spiele und die anderen Medien werden heute durch Downloads vertrieben. Die aktuellen Versionen verfügen über einen Flashspeicher und eignen sich nicht nur fürs Spielen bzw. den Konsum von Filmen und Musik. Man kann mit der PSP beispielsweise auch im Internet surfen oder sich mit anderen Gamern über das Playstation Network vernetzen. Die PSP eignet sich auch als Medienplayer. Hierfür schließt man die PSP einfach an den TV an. Auch sind einzelne PSPs über eine Playstation 3 miteinander vernetzbar.

\section{Playstation Vita}

Die Playstation Vita ist der Nachfolger der PSP und verfügt über eine Vielzahl an Funktionen sowohl im Bereich Gaming als auch für den Medienkonsum oder die Vernetzung der Spieler untereinander. Das Modell ist keine Weiterentwicklung der PSP sondern etwas komplett Neues. Die Vita verfügt über einen hochauflösenden Touchscreen, 
mehrere Spieltasten, zwei Kameras, Mikrofon, Lautsprecher, Wifi, auf Wunsch 3G, Bewegungssteuerung und ein weiteres Touchpad auf der Unterseite. Die Nutzungsmöglichkeiten in Spielen sind beeindruckend. Die vielen Controllerfunktionen erzeugen ein völlig neues Spielgefühl. Die Vita ermöglicht auch das Spielen von Games, die auf der Playstation 3 laufen. Das bedeutet, man spielt mit der Vita gegen Spieler, die das gleiche Spiel auf der Playstation 3 spielen. Die Kamera auf der Rückseite ermöglicht sogenannte Augmented Reality-Spiele. Hierbei wird z. B. das Spiel „Reality Fighters“ in die reale Welt übertragen. Ich schaue also auf das Display und die Kamera zeigt mir auf dem Display meine reale Umgebung. Nun wird das Spiel in diese Umgebung projiziert. Das Mikrofon ermöglicht eine Unterhaltung mit anderen Spielern sowie die Steuerung ausgesuchter Funktionen in bestimmten Spielen. Natürlich ist auch diese Konsole an das Playstation-Network angeschlossen. Schließlich kann man sich direkt mit anderen Vita-Besitzern vernetzen und gegeneinander spielen. Mittels GPS kann ich wiederum ortsbasierte Community-Funktionen sowie Spiele nutzen.

\section{Zusammenfassung}

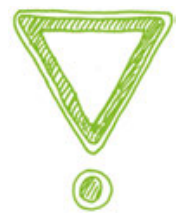

Die mobilen Konsolen haben sich kontinuierlich von reinen Gaming-Plattformen zu multioptionalen Medienplattformen verändert. Im Gegensatz zu z. B. Smartphones haben wir es hier aber mit Systemen zu tun, die speziell für den Bereich Gaming entwickelt wurden. Durch Anbindung an das Internet und die damit verbundenen zusätzlichen Services wie z.B. die Communityfunktionen, sind die aktuellen Systeme eine direkte Konkurrenz in Sachen Gaming zu den Smartphones. Trotzdem zeichnet sich ab, dass die Smartphones diesen Konkurrenzkamp gewinnen werden.

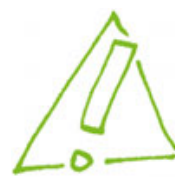

Auch bei diesen Plattformen ist der erste Schritt das Ausprobieren der Geräte, damit möglichst viele Mitarbeiter in der Lage sind, in diesem Bereich aktiv zu werden.

\section{Tablets und Smartphones}

Mit den Tablets und den Smartphones begeben wir uns nun in eine Welt von Geräten, die nicht primär für das Spielen von Computergames entwickelt wurden. Im Folgenden werde ich auf die Besonderheiten dieser Hardware eingehen. Bezüglich der Software werde ich mich in einem späteren Kapitel äußern.

Wie bereits erwähnt, handelt es sich bei dieser Hardware um Systeme, die nicht speziell für das Thema Gaming entwickelt worden sind. Das bedeutet, dass ihre Funktionen im Vergleich zu Gaming-Systemen vor allem den mobilen Angeboten wie dem Nintendo 3DS oder der Playstation Vita beschränkt sind. Die Spiele müssen über die jeweiligen Touchscreens gesteuert bzw. gespielt werden. Dies erfordert ein Umdenken in der Spieleentwicklung. Gleichzeitig verfügen diese Systeme über andere Funktionen bzw. werden bestimmte Funktionen anders genutzt.

Gaming auf mobilen Kommunikationssystemen ist kein neuer Trend. Schon die ersten massenkompatiblen Handys besaßen rudimentäre Spiele wie z. B. „Snake“ oder „Minesweeper“. Die Spiele wurden über die Tastatur gespielt und waren dementsprechend schwierig. Sie waren eher ein nettes Beiwerk aber nicht mehr. Erst mit der Entwicklung der Smartphones und Tablets kam die Gamesentwicklung in diesem Sektor in Schwung. Viele Menschen nutzten bis dahin ein Handy für die Kommunikation und eine mobile Gaming-Konsole um zu spielen. Mit dem Erscheinen des 
iPhones (bzw. kurz davor dem iPod) sowie den weiteren Angeboten anderer Hersteller wurde Gaming auf Smartphones relevant. Dies hatte verschiedene Gründe:

1. Aktuelle Smartphones sind kleine Computer. Sie verfügen über eine sehr gute Grafikleistung, starke Prozessoren, große Arbeitsspeicher und sehr gute Touchscreen-Displays. Es lassen sich auch komplexe Spiele und Darstellungen realisieren. Wahrscheinlich sind es bei den weitaus meisten Smartphones die Games, die die technischen Möglichkeiten des Systems wirklich ausreizen.

2. Die Hersteller der Smartphones kommen aus Gesellschaften in denen Entertainment und/oder Gaming eine große Rolle spielen.

3. Die Gaming-Industrie sucht händeringend nach neuen Geschäftsmodellen. Dies betrifft sowohl große Konzerne als auch die sogenannten „Independent Entwickler“. Für beide Gruppen gilt: Der rein digitale Vertrieb ermöglicht es jedem Entwickler und jedem Unternehmen, seine Inhalte anzubieten. Der teure Flaschenhals des stationären Handels entfällt.

4. Die Technologien vor allem der Smartphones ermöglichen völlig neue Spiel- bzw. Geschäftsmodelle.

\section{Betriebssysteme}

Sowohl im Bereich der stationären Konsolen als auch bei den mobilen Konsolen gibt es verschiedene Anbieter und damit verbunden verschiedene Produkte. Bezogen auf Smartphones und Tablets ist die Situation ähnlich. Allerdings stellt dies kein größeres Problem dar. Zwar gibt es eine sehr große Zahl an verschiedenen Geräten. Jedoch ist das Grundmodell bei allen Geräten gleich. Die Unterschiede liegen in den technischen Möglichkeiten, also z. B. in der Frage wie groß der Arbeitsspeicher ist, oder wie gut das Display funktioniert, und natürlich im Betriebssystem. Zum jetzigen Zeitpunkt gibt es nur zwei relevante Betriebssysteme in diesem Bereich: Android und iOS.

Android ist das Betriebssystem von Google. Dieses System ist nicht an eine bestimmte Hardware gebunden. Zwar verkauft Google mit dem NEXUS ein eigenes Smartphone, jedoch steht es auch anderen Anbietern frei, das Betriebssystem zu nutzen. Somit konnte sich das Betriebssystem erfolgreich verbreiten. Heute ist Android das weltweit meist genutzte Betriebssystem. Android wird sowohl aus Smartphones als auch auf Tablets genutzt.

Die Nutzung eines Betriebssystems sowohl auf Smartphones als auch auf Tablets ist auch beim zweiten Betriebssystem gegeben: iOS. Dabei handelt es sich um das Betriebssystem von Apple. Im Gegensatz zu Android ist iOS an die Geräte des Unternehmens (iPad und iPhone) gebunden.

Zwar gibt es noch weitere Betriebssysteme anderer Anbieter wie z.B. Windows Phone, Windows 8 (Microsoft), Firefox (Mozilla Foundation), Blackberry (RIM) etc. Jedoch spielen diese im Moment keine relevante Rolle. Auch wenn Windows 8 an sich ein spannendes System ist.

Bei Android und iOS werden die Spiele über einen Online-Store bezogen. Bei iOS heißt dieser „App-Store“ und bei Android „Google Play“ Es ist mehr als interessant, dass Google für seinen Onlinestore das Wort „Play“ benutzt. 


\section{Gaming auf Smartphones und Tablets}

Es gibt zwei Arten von Spielen:

1. Spiele, die bereits auf anderen Plattformen veröffentlicht wurden und nun für Smartphones und/oder Tablets adaptiert werden

2. Spiele, die speziell für Smartphones und/oder Tablets entwickelt werden

Natürlich gibt es auch Spiele, die ursprünglich für Smartphones und Tablets entwickelt und dann für andere Plattformen wie z. B. dem PC adaptiert worden sind.

Bei den unter Punkt 2 genannten Spielen sind vor allem die Spiele spannend, die mehr sind als Videospiele für Mobile Devices. Einige dieser Spiele integrieren z. B. die reale Welt in das Spiel.

Da Smartphones und Tablets keine Tasten haben, über die man das Spiel kontrollieren kann, findet die Steuerung über den Touchscreen statt. In vielen Fällen wird auf dem Touchscreen ein Controller simuliert. Natürlich gibt es auch eine klassische Touchscreen-Steuerung. In diesen Fällen werden z. B. Gegenstände durch Bewegungen auf dem Touchscreen bewegt. Darüber hinaus gibt es Spiele, bei denen die Bewegungssensoren genutzt werden. Bei manchen Autorennen hält man beispielsweise das ganze iPad wie ein Lenkrad und steuert das Auto durch die Bewegungen des ganzen Gerätes. Es gibt auch Flugsimulationen bei denen sowohl durch die horizontale als auch durch die vertikale Bewegung das Fluggerät gesteuert wird.

Neben der Steuerung werden auch Kamerasysteme in die Spiele integriert. So werden z. B. bei dem Spiel „Ghostbusters“ die einzufangenden Geister in die reale Umgebung projiziert. Auch die Möglichkeit der GPS-Ortung wird in Spiele integriert, nämlich dann wenn lokale Orte in der realen Welt Teil des Spieles sind. Beim Spiel „Ingress“ z. B. werden reale Orte als Portale genutzt. Um ein Portal zu finden muss man in der realen Welt den dazugehörigen Ort finden. In diesem Fall funktioniert dies über eine Einbindung von Google-Maps. Die Kameras ermöglichen auch die Einbindung von QR-Codes und natürlich von Sozialen Netzwerken wie Facebook, Google+ oder Twitter.

\section{Trends}

Mit den Trends im Gaming-Sektor ist es immer so eine Sache. Die Gaming-Welt verändert sich stetig und mit hoher Geschwindigkeit. Wie also soll ich heute wissen, was in fünf Jahren relevant ist? Insofern ist es unmöglich, hier genaue Aussagen zu treffen. Andererseits ist es so, dass wir bestimmte Veränderungen im Mediennutzungsverhalten sowie in der Nutzung von Kommunikationstechnologien beobachten können. Daraus ergeben sich bestimmte Muster. Zugleich können wir sehen, welche Veränderungen im Bereich Hard- und Software stattfinden. Aus diesen Informationen kann man zumindest Wahrscheinlichkeiten benennen. Ziel solcher Aussagen ist dann auch, dass man sich mit den Themen und Inhalten beschäftigt. Es geht also darum, Sie auf bestimmte Phänomene hinzuweisen. In diesem Trend-Block geht es um die Hardware und damit verbunden um mögliche Veränderungen in der Zukunft.

Im Bereich des mobilen Gaming werden vor allem Smartphones eine sehr große Rolle spielen. Inwieweit sie mobile Gaming-Konsolen wie z. B. den Nintendo 3DS oder die Playstation Vita verdrängen, bleibt abzuwarten. Smartphones sind ideale Gaming-Maschinen - vor allem, da Sie eine Vielzahl an Zusatzfunktionen mitbringen, die sich in das Gameplay integrieren lassen. Andererseits verfügen Sie nicht über die 
Controller-Funktionen einer mobilen Konsole. Klar ist aber, dass die Vertriebsmodelle so oder so digital sein werden. Hier wird es für Bibliotheken besonders schwer werden, eine klassische Bestandsarbeit zu realisieren.

Stationäre Konsolen entwickeln sich immer mehr zu multioptionalen Medienplattformen. Das bedeutet, dass diese Systeme in Zukunft zu direkten und starken Konkurrenten von Bibliotheken werden. Vor allem dann, wenn die Film- und Musikindustrie ihre digitalen Angebote zu Lasten der analogen Angebote ausbauen wird.

Die Playstation 4 und die XBOX ONE werden die technologischen Möglichkeiten noch einmal massiv erweitern. Der Gaming-PC wird wahrscheinlich in zwei Formen vorhanden sein. Da wird es zum Einen die hochgerüsteten Gaming-PCs geben, die mit teuren Grafikkarten, speziellen Tastaturen und Mäusen völlig neue Spielformen ermöglichen. Diejenigen, die sich ein solches System anschaffen, werden damit vor allem komplexe und grafisch aufwendige Spiele spielen wollen. Gleichzeitig wird der Standart-PC eine Aufwertung erleben. Viele Spiele, gerade in den Bereichen Onlinegaming und Social-Gaming benötigen keine hohe Rechenleistung, da diese Spiele online im Internet-Browser gespielt werden. Wir können aber auch beobachten, dass der Markt für Heimcomputer kontinuierlich schrumpft. Viele Menschen nutzen anstatt eines Computers eher ein Tablet. Hier könnte es also zu weitreichenden Veränderungen kommen. Gleichwohl bedeuten auch Onlinegaming und Gaming auf Tablets eine neue Herausforderung für Bibliotheken, weil diese Games nicht mehr Teil des Bestands werden können. 